UNITED STATES DEPARTMENT OF THE INTERIOR

GEOLOGICAL SURVEY

\begin{abstract}
Tertiary Foraminiferal Localities in the Cape Flattery Area, Northwestern Olympic Peninsula, Washington
\end{abstract}

P. D. Snavely, Jr. ${ }^{1}$, W. W. Rau ${ }^{2}$, and D. J. Hafley ${ }^{1}$

Open-File Report 86-344A

This report is preliminary and has not been reviewed for conformity with U.S. Geological survey editorial standards and stratigraphic nomenclature.

1. U.S. Geological survey, Menlo Park, California
2. Washington State Division of Geology and Earth Resources, olympia, washington 


\section{Tertiary Foraminiferal Localities in the Cape Flattery Area, Northwestern Olympic Peninsula, Washington}

by P. D. Snavely, Jr. ${ }^{1}$, W. W. Rau ${ }^{2}$, and D. J. Hafley ${ }^{1}$

The biostratigraphic framework for the Tertiary strata mapped by Snavely and others (1986) in the Clallam Bay, Cape Flattery, Lake Pleasant, and Ozette Lake 15-minute quadrangles is based chiefly upon age determinations made by Rau (see Snavely and others, 1980) from his study of foraminifers. Although these mainly bathyal Tertiary sedimentary rocks generally contain sparse foraminifers, they indicate that the rocks range in age from early Eocene to early Miocene. Most of the samples were collected along river beds or in logging road cuts where the rocks are the least weathered. Nevertheless, most of the samples collected south of the Crescent Thrust Fault were barren, as these strata are structurally complex and have suffered secondary alteration.

The foraminiferal slides prepared by Rau for this area are part of the U.S. Geological Survey, Pacific Northwest Foraminiferal Reference Collection which presently is housed in the Division of Geology and Earth Resources, Olympia, Washington. Rau's assignments of foraminiferal assemblages to Eocene stages of Mallory (1959), Eocene and Oligocene stages of Schenck and Kleinpell (1936), and Oligocene and Miocene stages of Kleinpell (1938) are contained in published and written communications during the period 1969 to 1985 .

Tables list the field and reference collection number for each sample and sample location by latitude and longitude. The tables are grouped under the new 1984 U.S. Geological Survey 7-1/2-minute quadrangles; the relationship of these quadrangles to the old 1956 and 1957, 15-minute quadrangles is indicated. The age and/or foraminiferal stage assignments by Weldon Rau are shown as well as the reliability of each age determination (call). Symbols listed under "call" are: G, good; F, fair; ?, questionable. The depositional environments (paleoenvironments) indicated by the foraminiferal assemblages are presented for most samples.

1. U.S. Geological Survey, 345 Middlefield Road, Menlo Park, California

2. Washington State Division of Geology and Earth Resources, Olympia, washington 


\section{REFERENCES CITED}

Kleinpell, R. M., 1938, Miocene stratigraphy of California: Tulsa, Oklahoma, American Association of Petroleum Geologists, 450 p.

Mallory, V. S., 1959, Lower Tertiary biostratigraphy of the California Coast Ranges: Tulsa, Oklahoma, American Association of Petroleum Geologists, $416 \mathrm{p}$.

Schenck, H. G., and Kleinpell, R. M., 1936, Refugian stage of the Pacific coast Tertiary: American Association of Petroleum Geologists Bulletin, v. 20 , no. 2 , p. $215-225$.

Snavely, P. D., Jr., Macleod, N. S., Niem, A. R., and Pearl, J. E., 1986, Geologic map of the Cape Flattery area, northwestern olympic Peninsula, washington: U.S. Geological Survey Open-File Report 86-344B (in press).

, Niem, A. R., Macleod N. S., Pearl, J. E., and Rau, W. W., 1980, Makah Formation--A deep-marginal-basin sequence of late Eocene and oligocene age in the northwestern Olympic Peninsula, Washington: U.S. Geological Survey Professional Paper 1162-B, 28 p. 


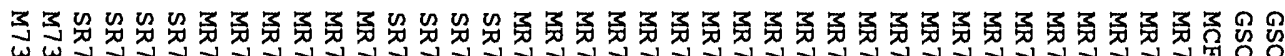

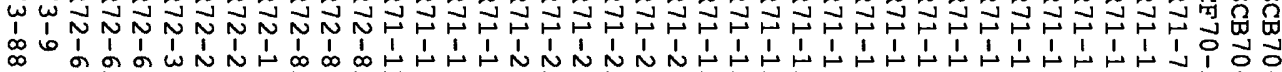

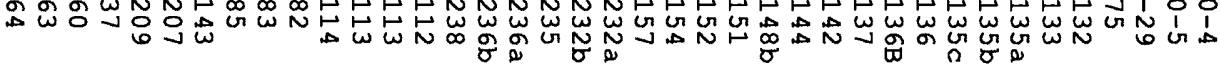

Јل

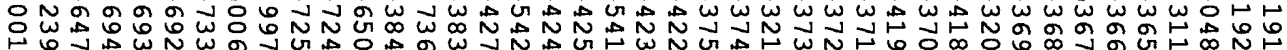

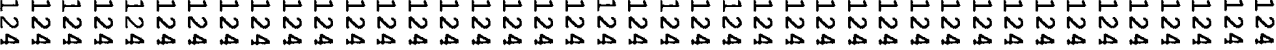

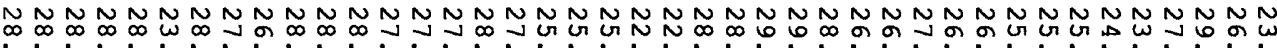
身

$\vec{\infty} \vec{\infty} \vec{\infty} \vec{\infty} \vec{\infty} \vec{\infty} \vec{\infty} \vec{\infty} \vec{\infty} \vec{\infty} \vec{\infty} \vec{\infty} \vec{\infty} \vec{\infty} \vec{\infty} \vec{\infty} \vec{\infty} \vec{\infty} \vec{\infty} \vec{\infty} \vec{\infty} \vec{\infty} \vec{\infty} \vec{\infty} \vec{\infty} \vec{\infty} \vec{\infty} \vec{\infty} \vec{\infty} \vec{\infty} \vec{\infty} \vec{\infty} \vec{\infty} \vec{\infty} \vec{\infty} \vec{\infty} \vec{\infty} \vec{\infty} \vec{\infty} \vec{\infty} \vec{\infty}$

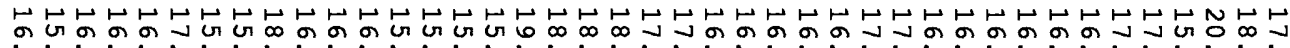

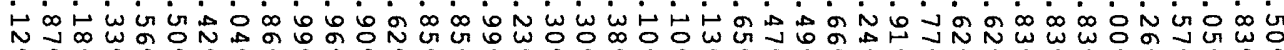

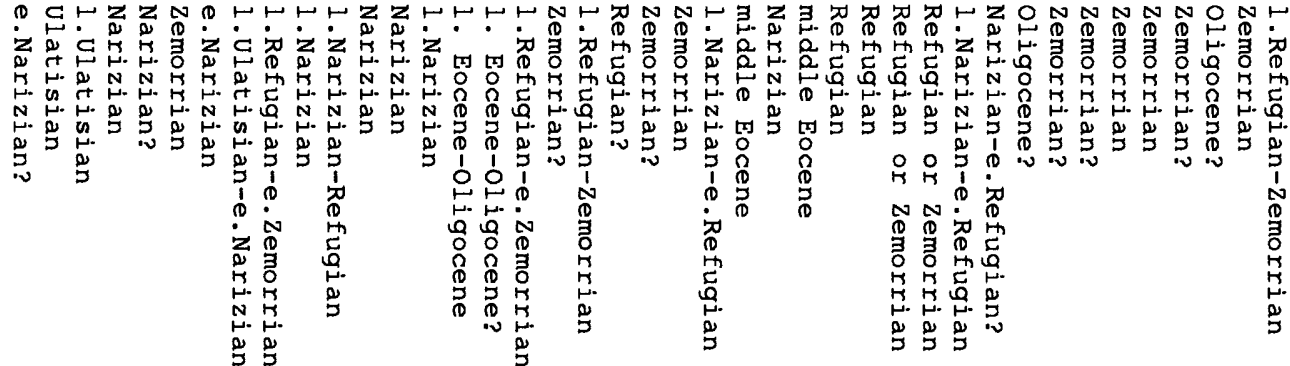

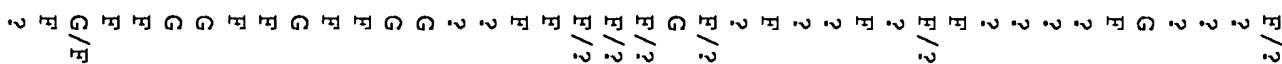

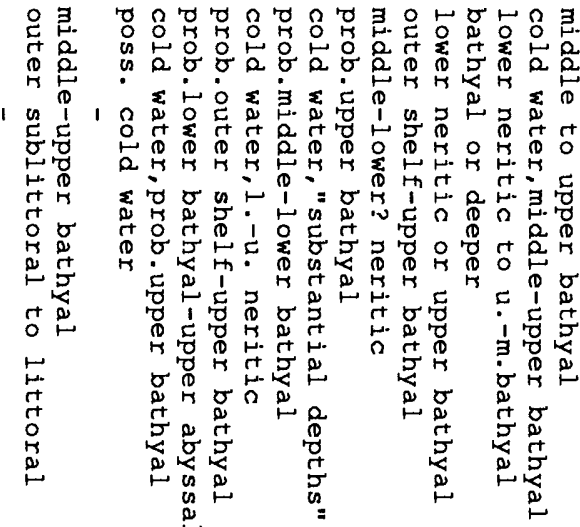

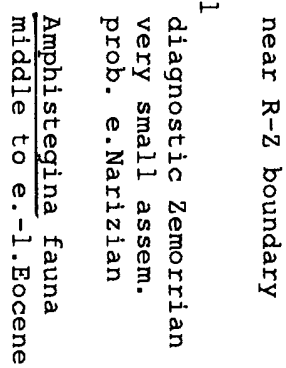

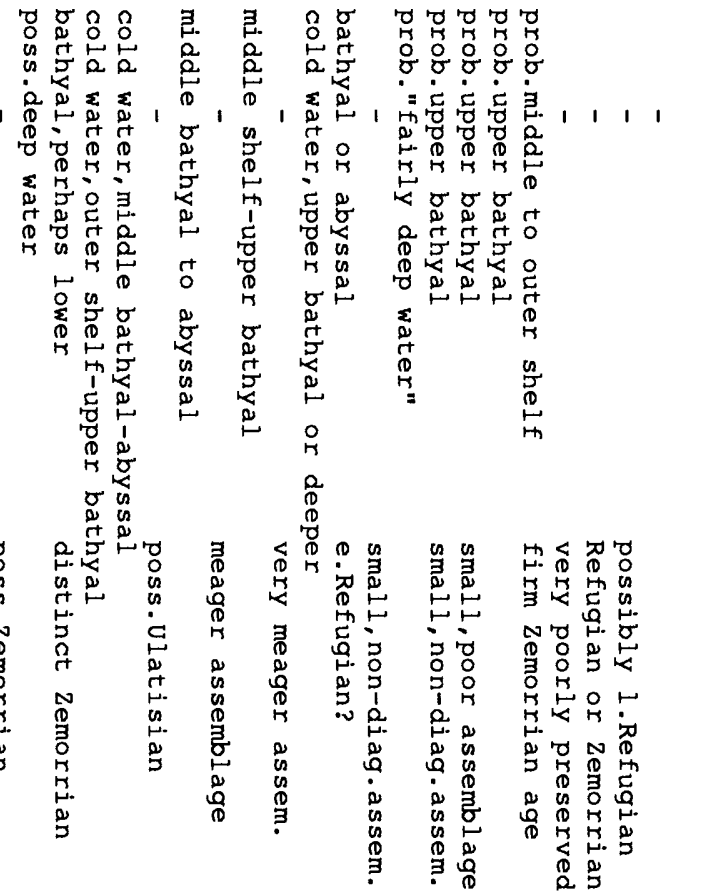




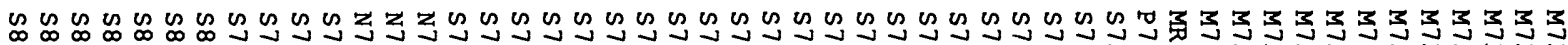
N

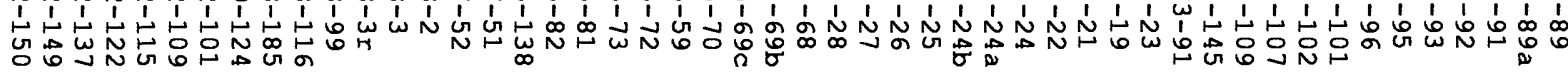

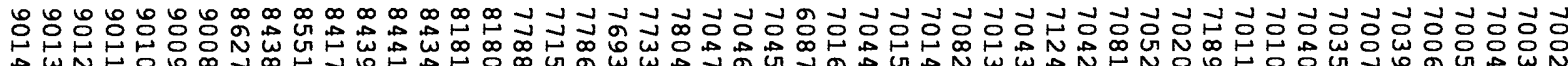

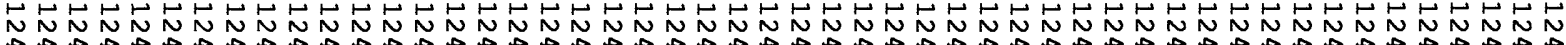

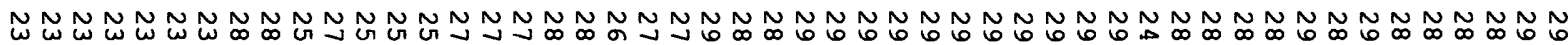

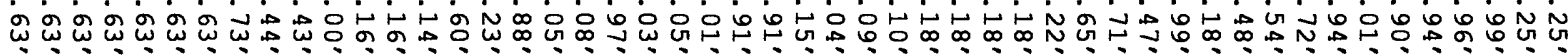

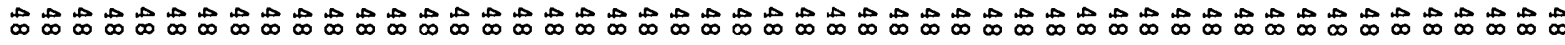

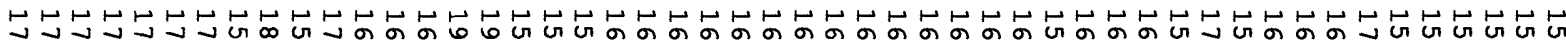

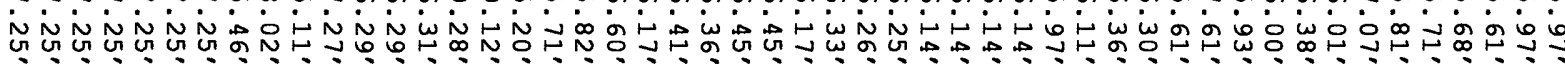

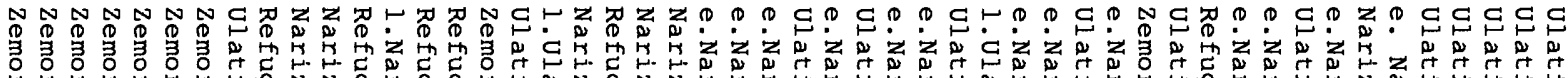

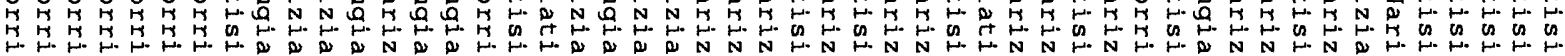

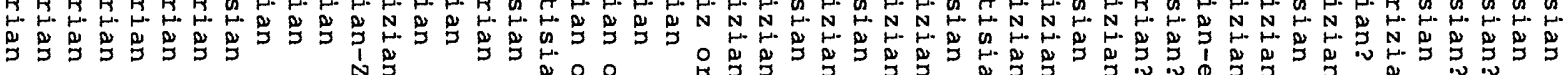

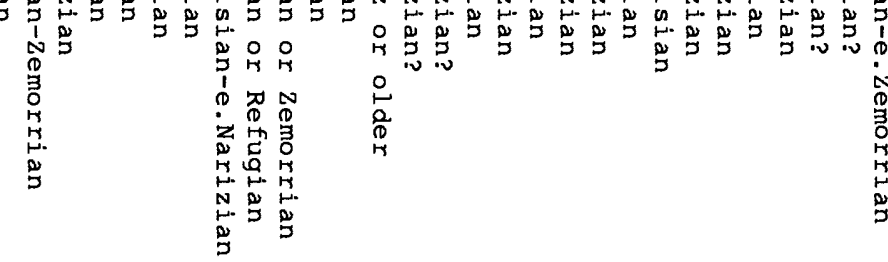

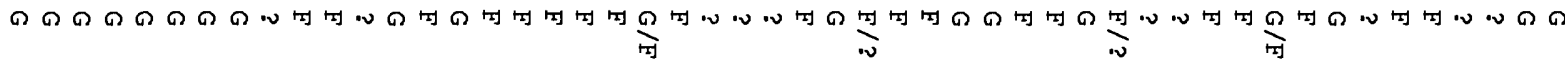

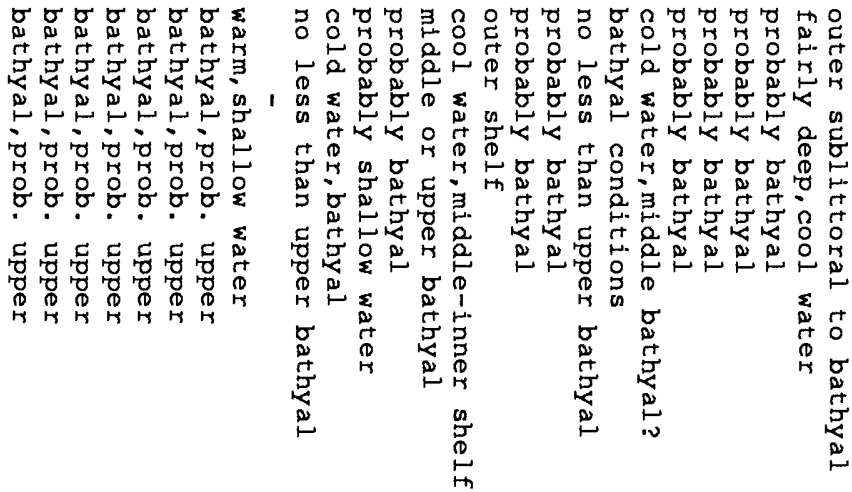

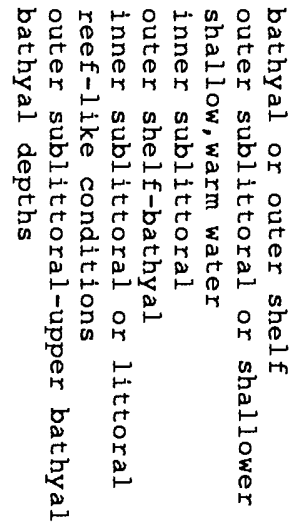

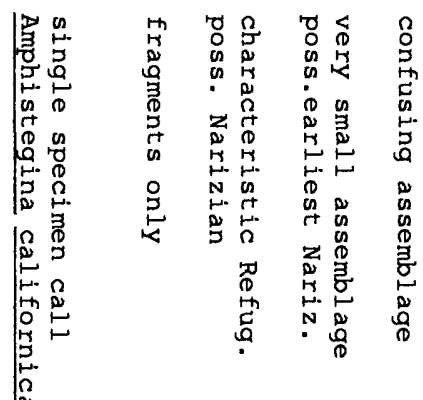

3. 'd

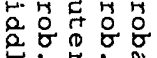

요 (1) 的占占

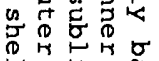

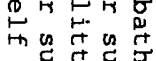

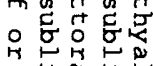

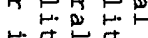

若贲苦

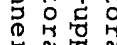

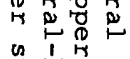

导宫

苗苦骂

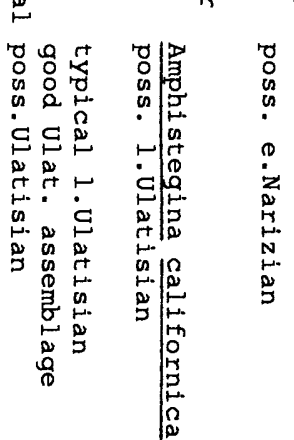




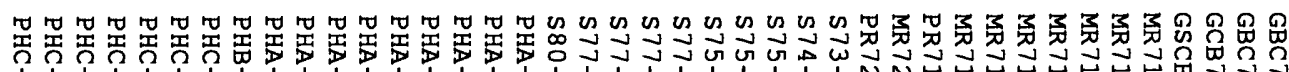

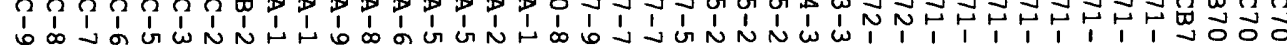

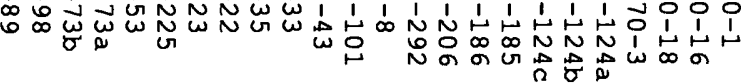

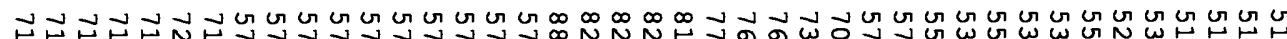

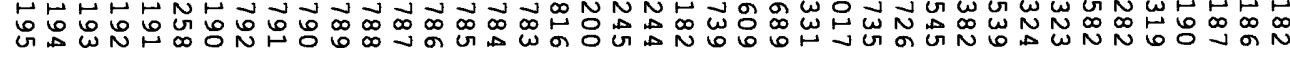

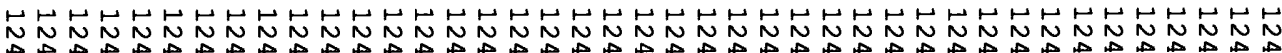
NNN

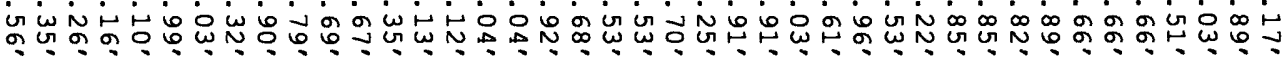

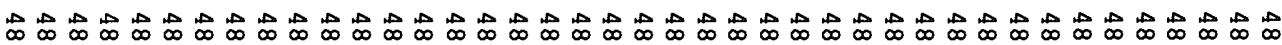
Є

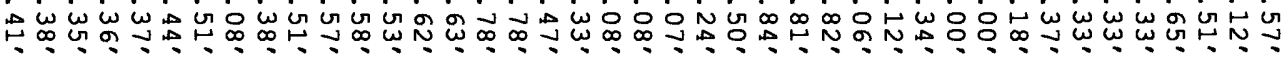

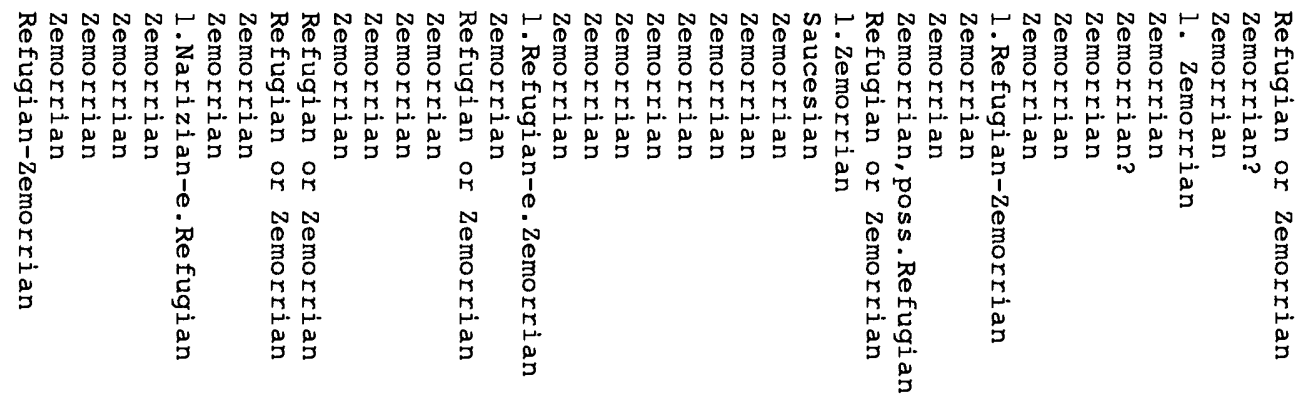

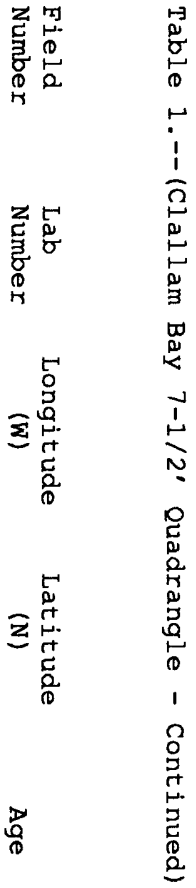

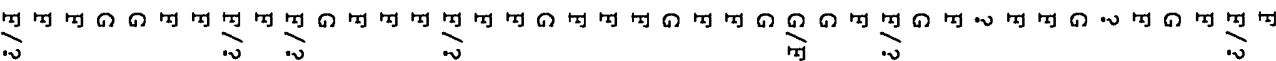

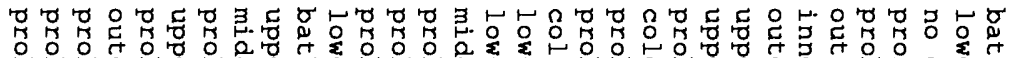

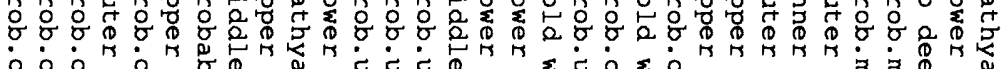

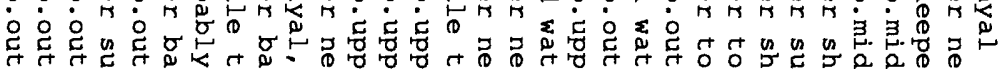

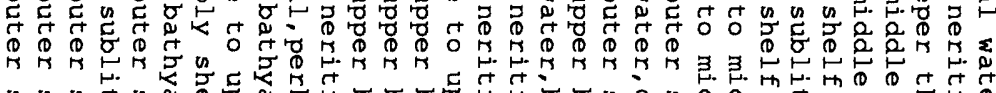

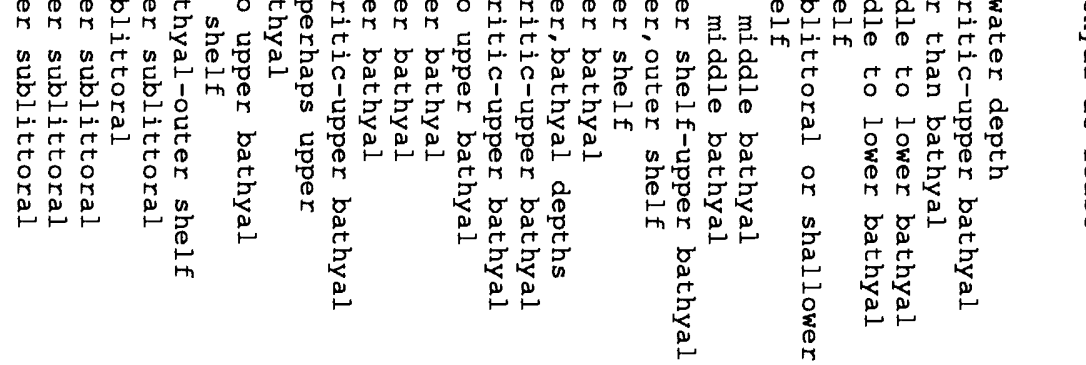

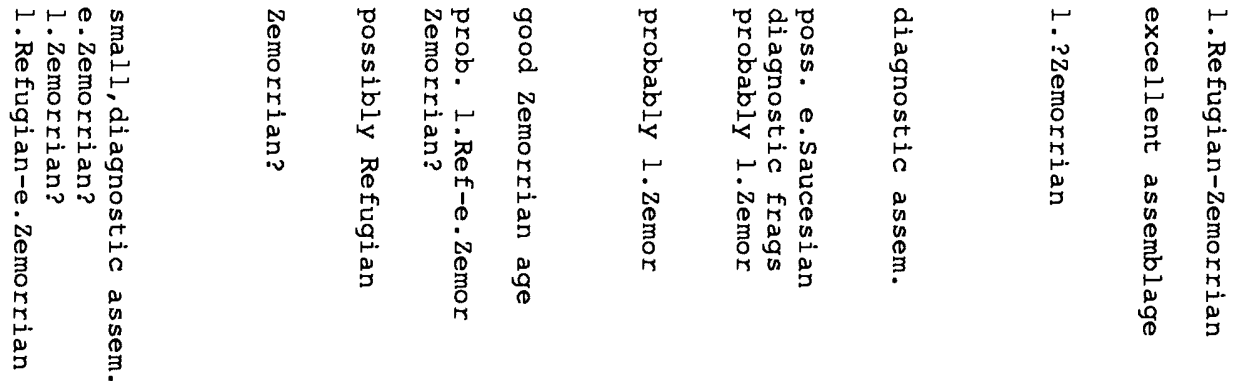




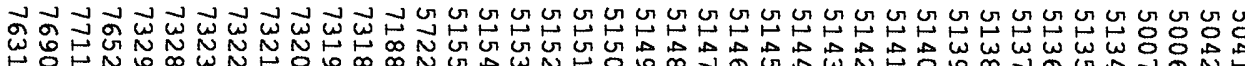

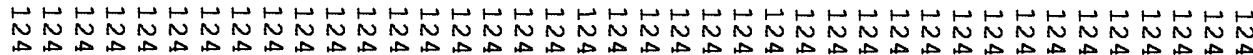

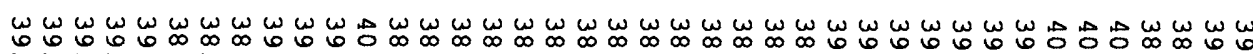
$\infty$ is ${ }_{0}$ on

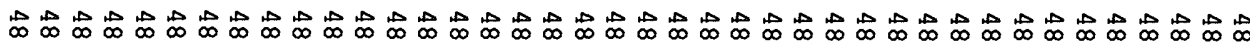

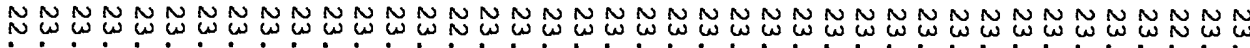

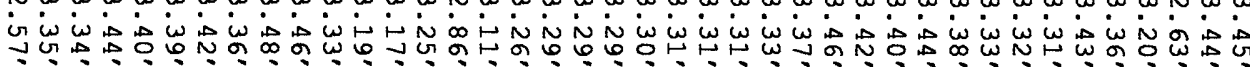

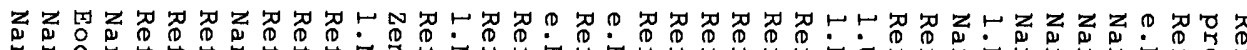

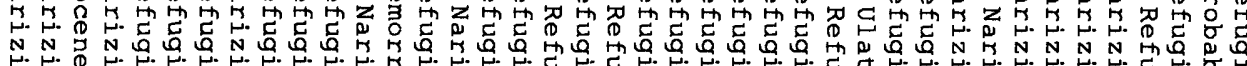

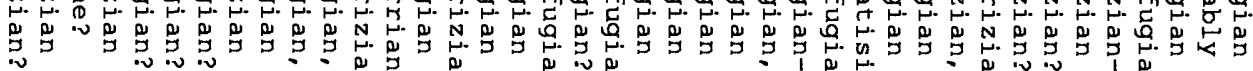

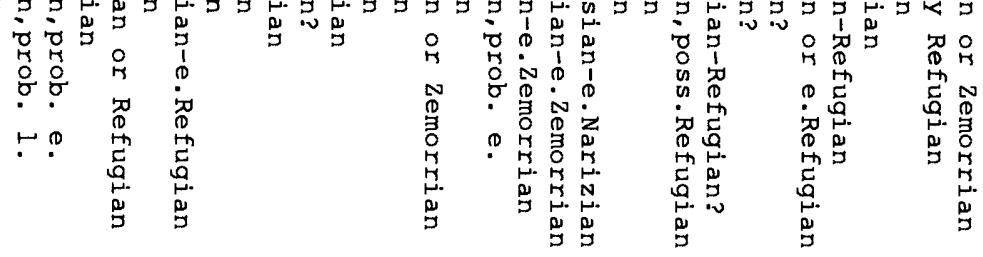

טיט

त्रो

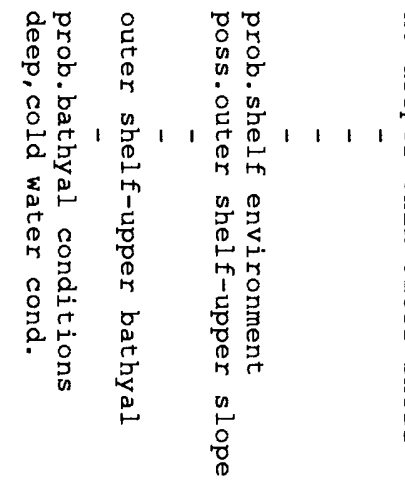

28

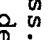

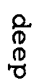

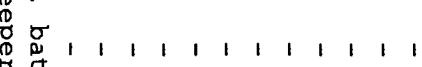

䓌点

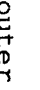

.

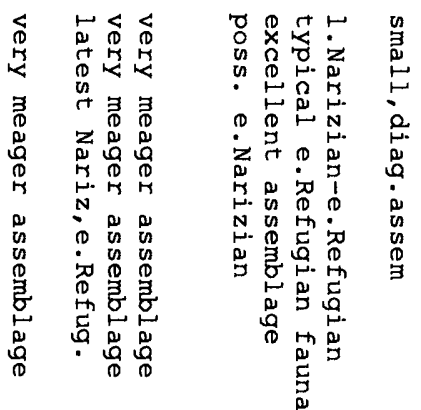

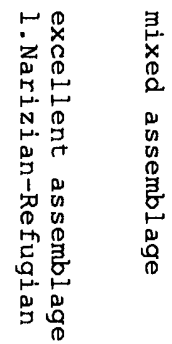

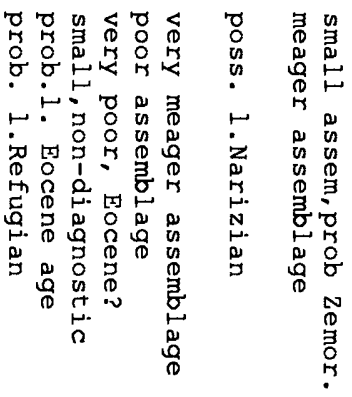

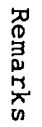




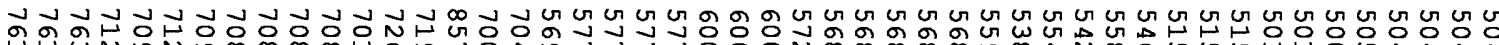

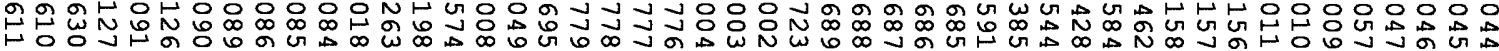

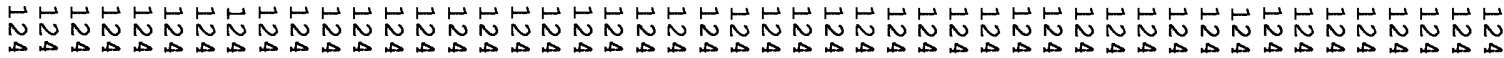

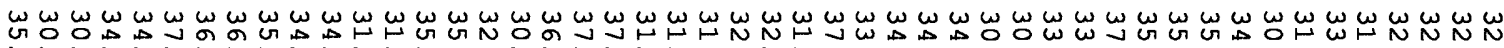

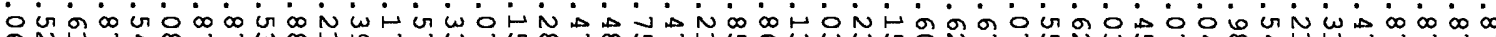

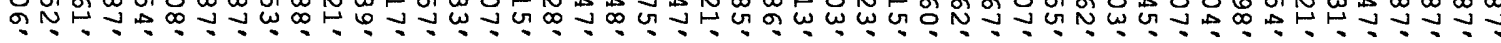

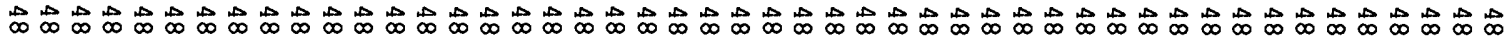
N

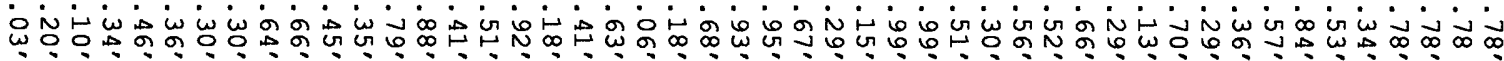

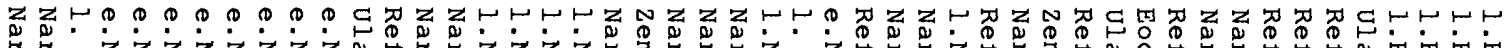
T.

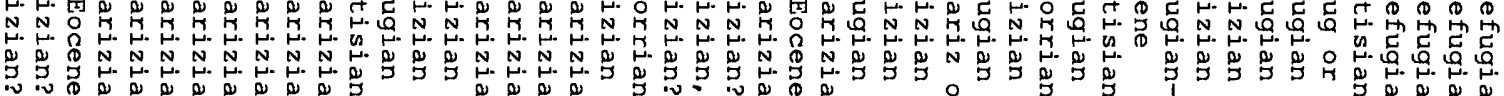

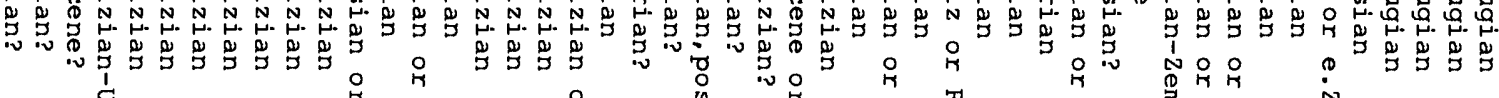

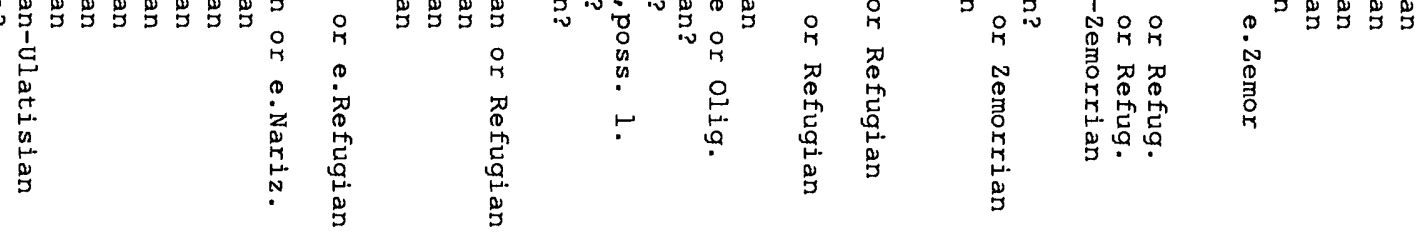

四四 ט

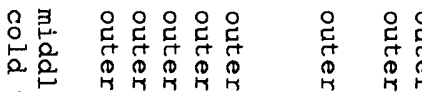

$\Sigma$

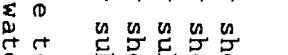

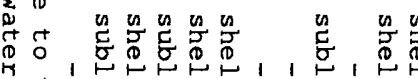

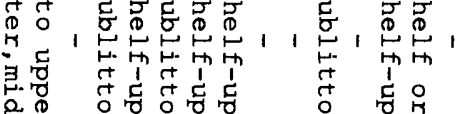

in

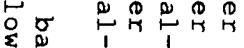

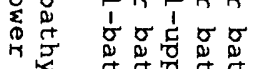

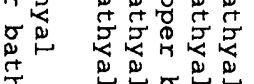

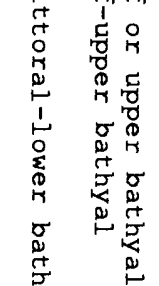

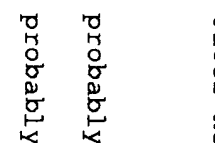

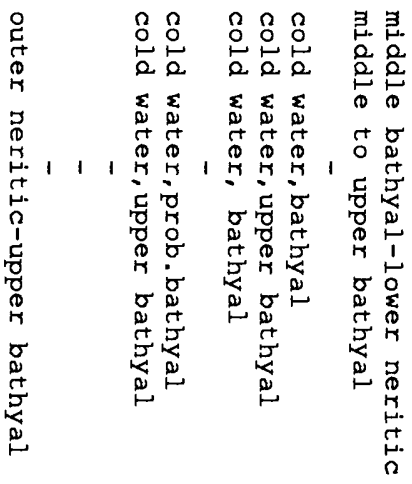

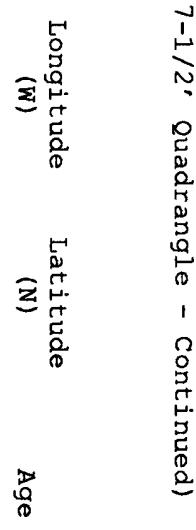

竞

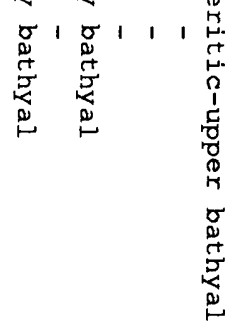

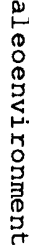

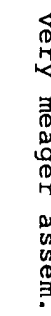

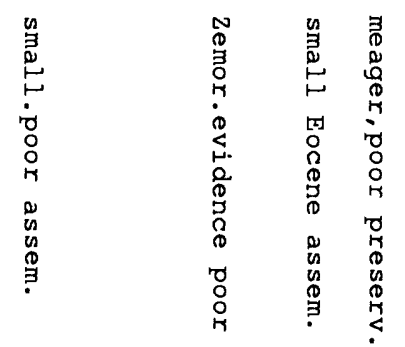

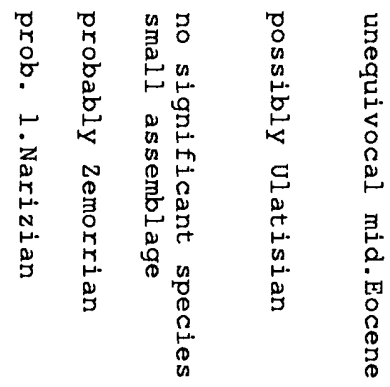

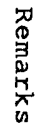




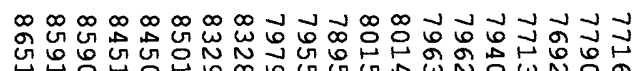
必

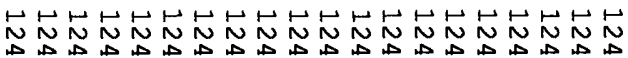

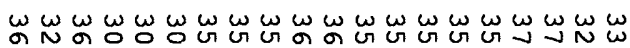

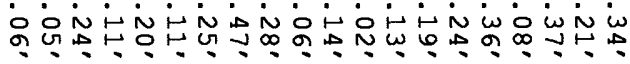

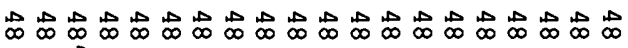

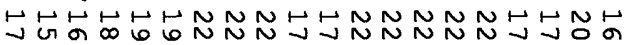

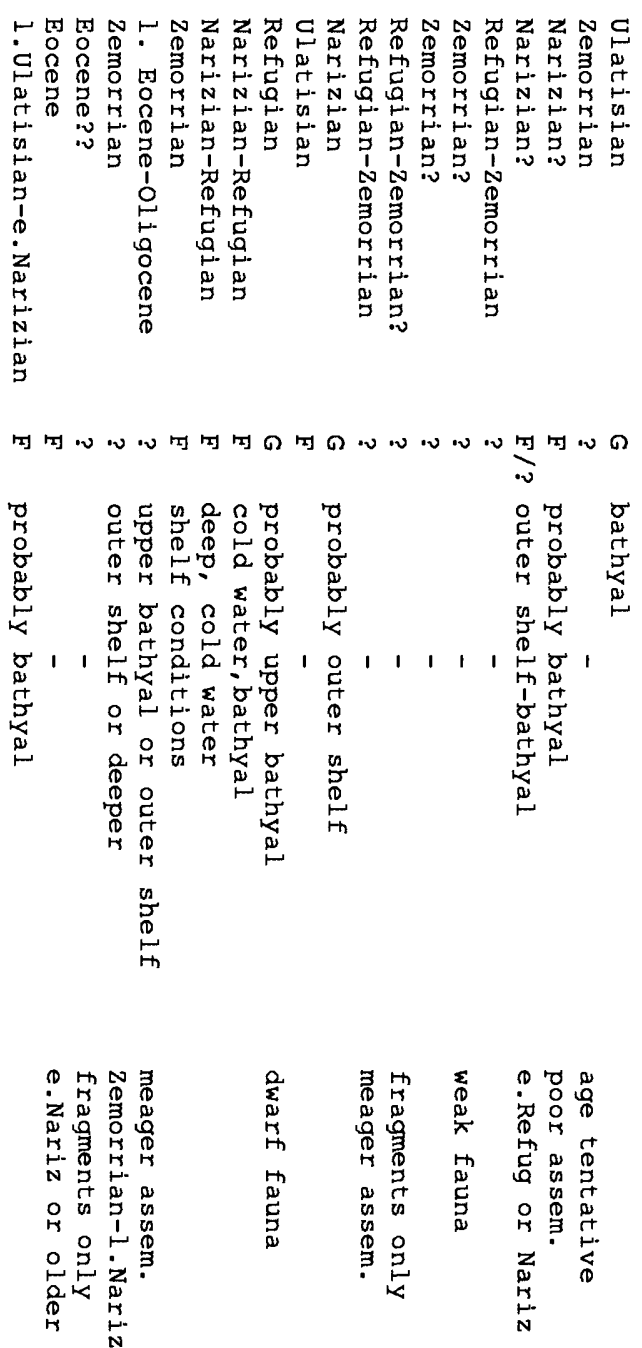


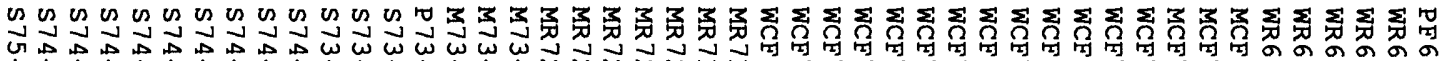

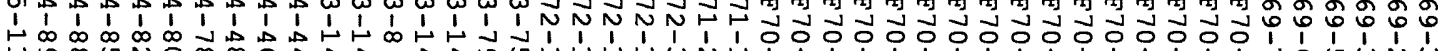
舟

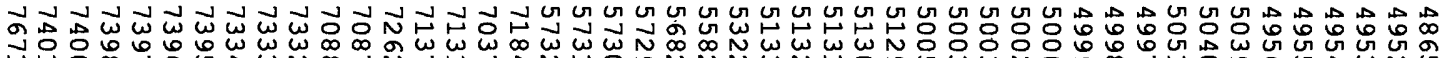

N

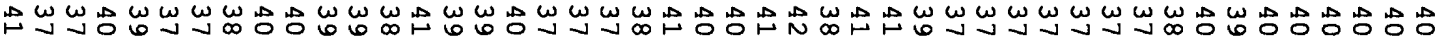

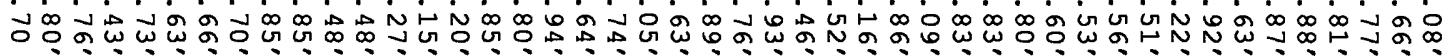

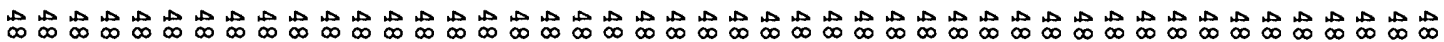

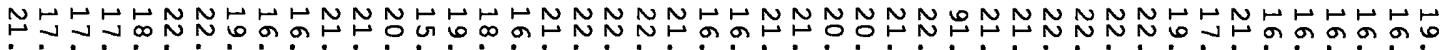

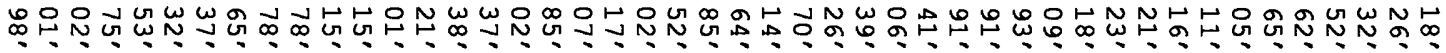

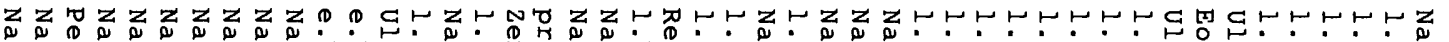

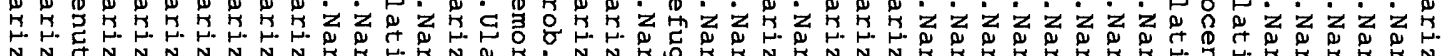
N. N.

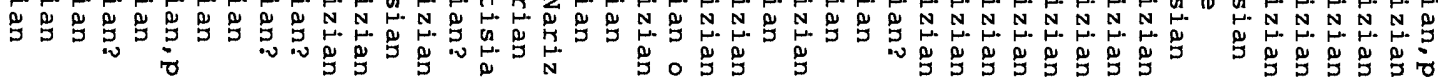

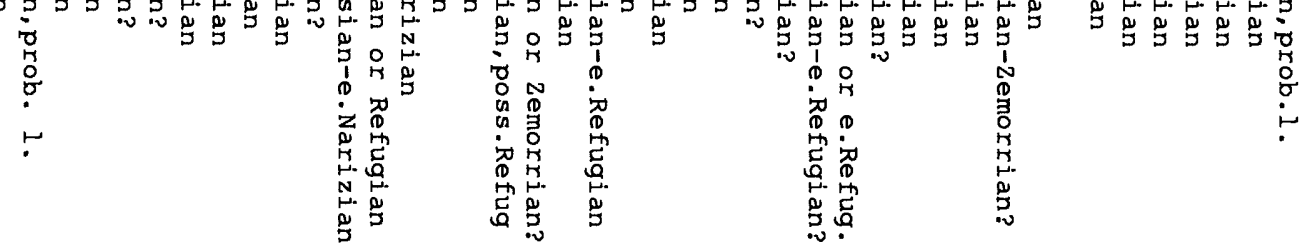

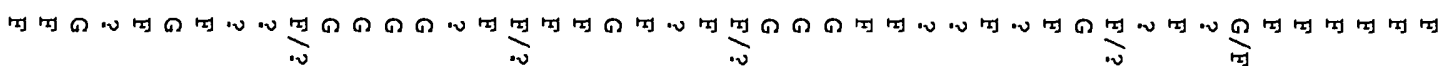

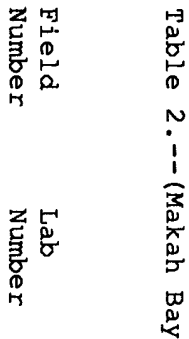

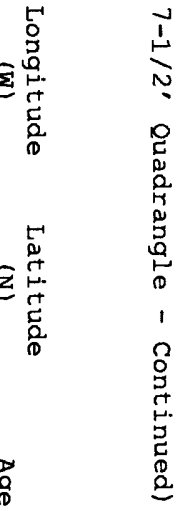

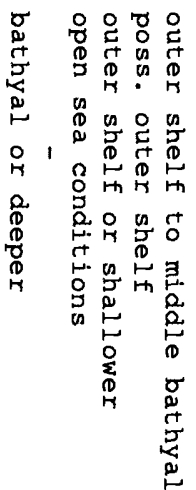

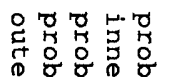

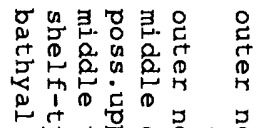

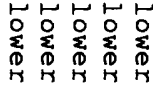

n 总芑员

б

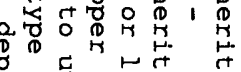

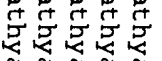

吕恕恕

ते

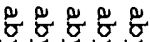

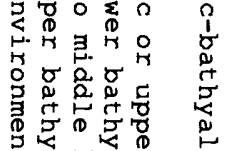

on on on

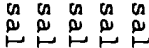

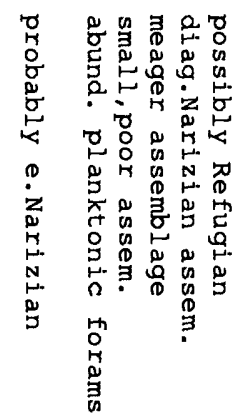

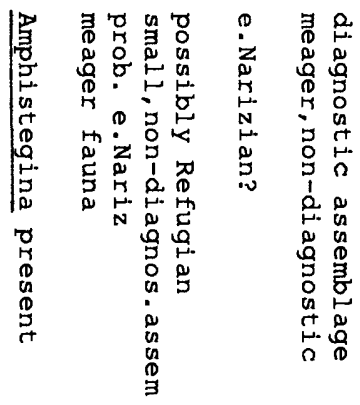

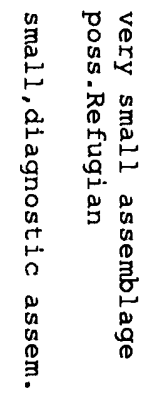

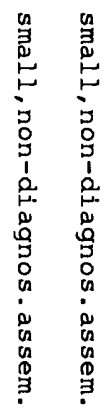

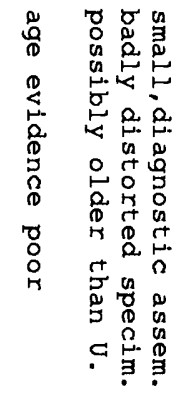

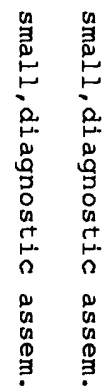

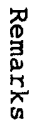




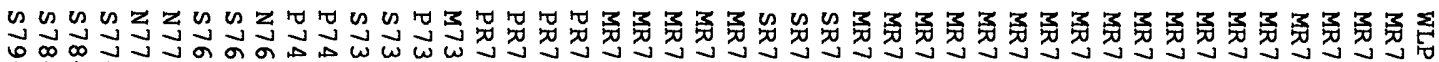

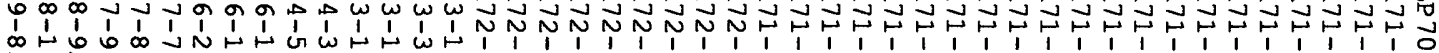

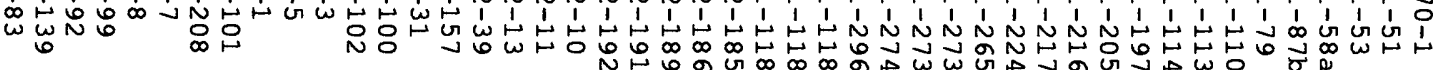

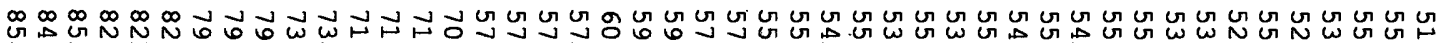

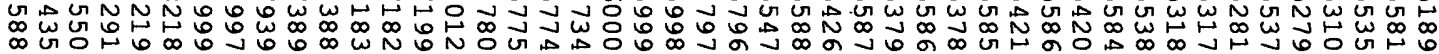

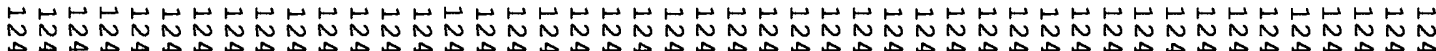
N N N

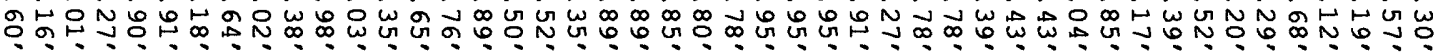

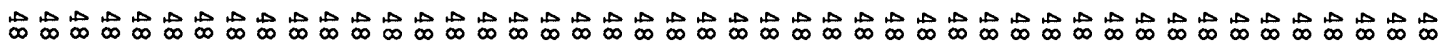

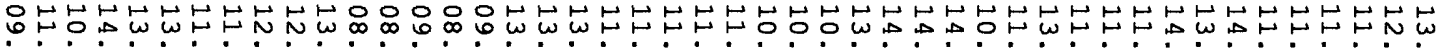

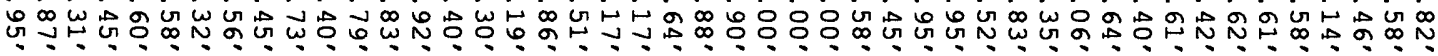

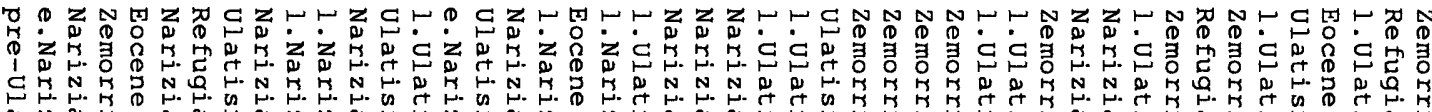

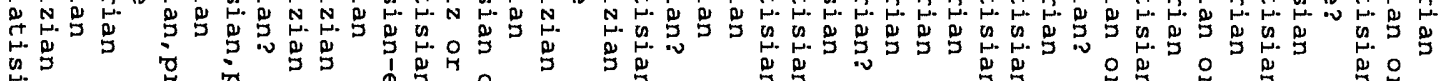

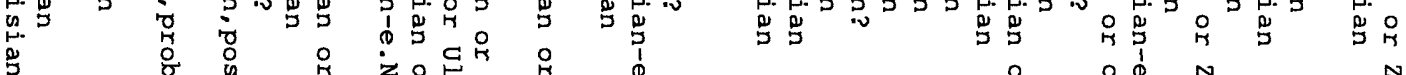

.

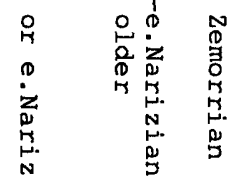

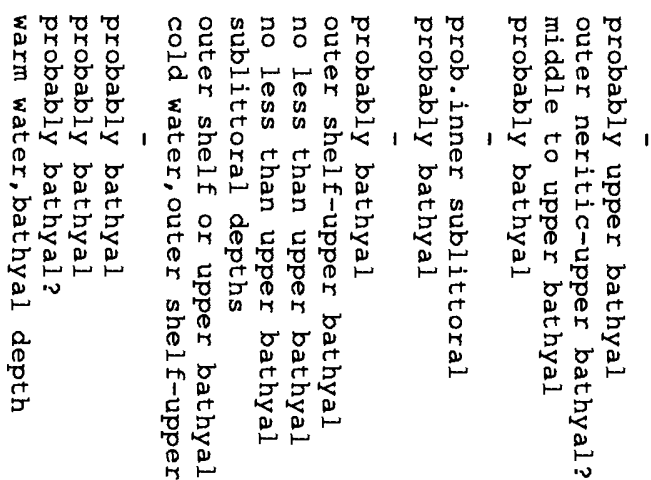

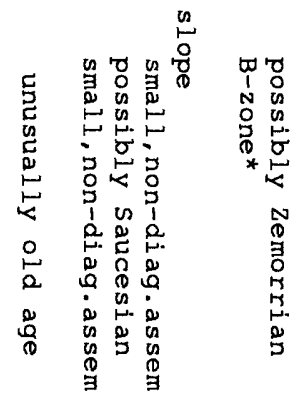

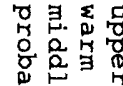

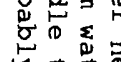

它。

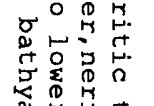

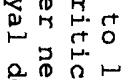

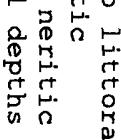

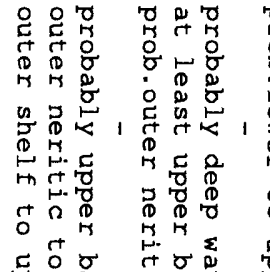

害骂骂

ช

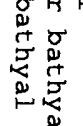

$\stackrel{2}{2}$

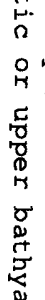
宫

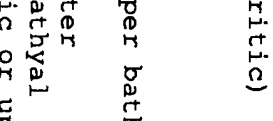

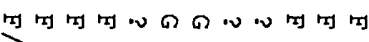

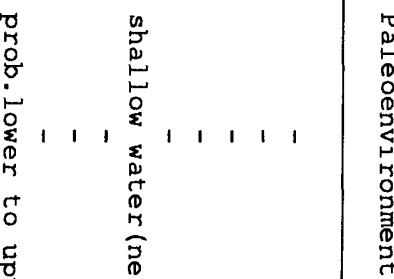

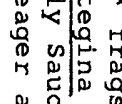

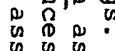

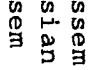




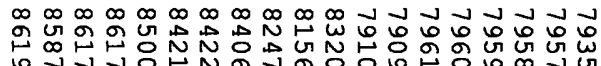

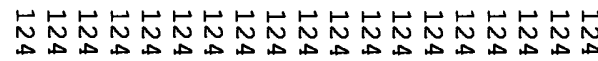

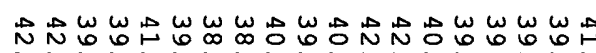

行宊

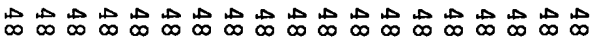

군

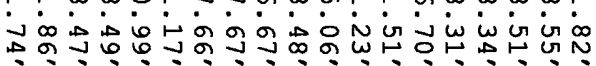

z

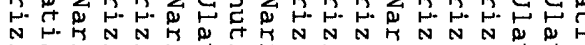

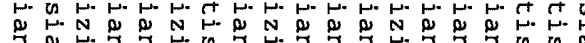

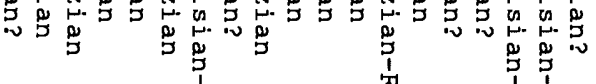

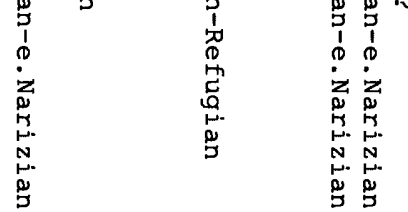

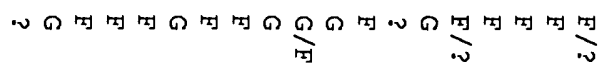
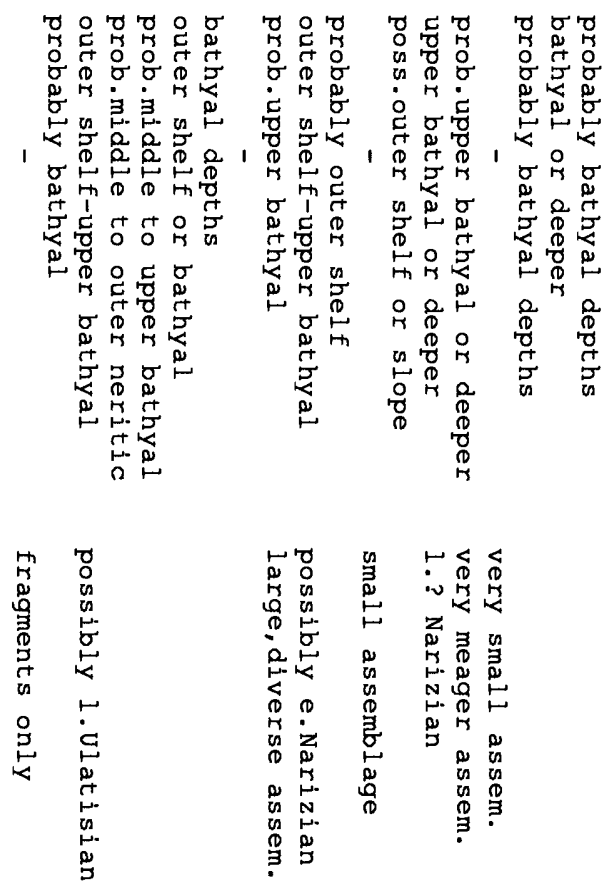


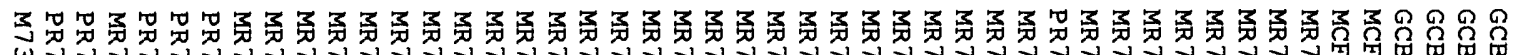

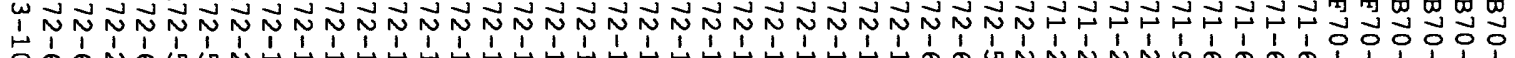

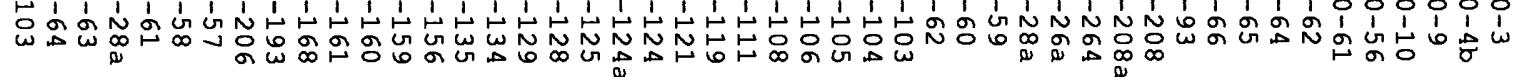

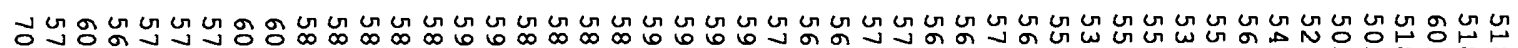

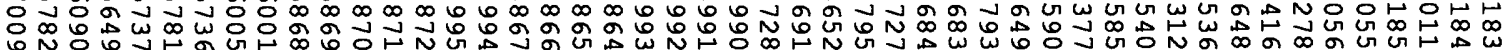

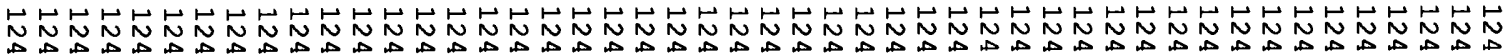

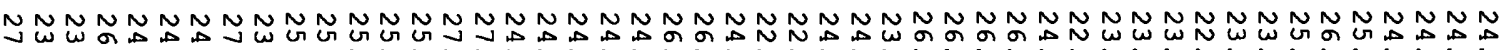
곡

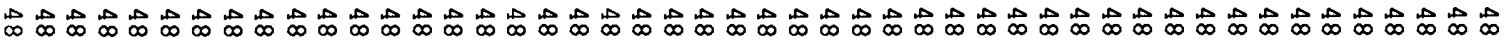

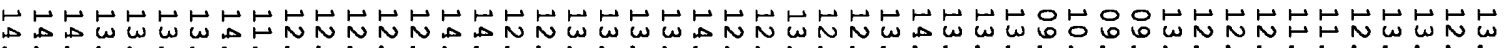

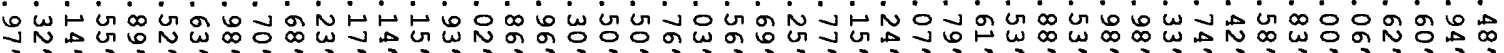

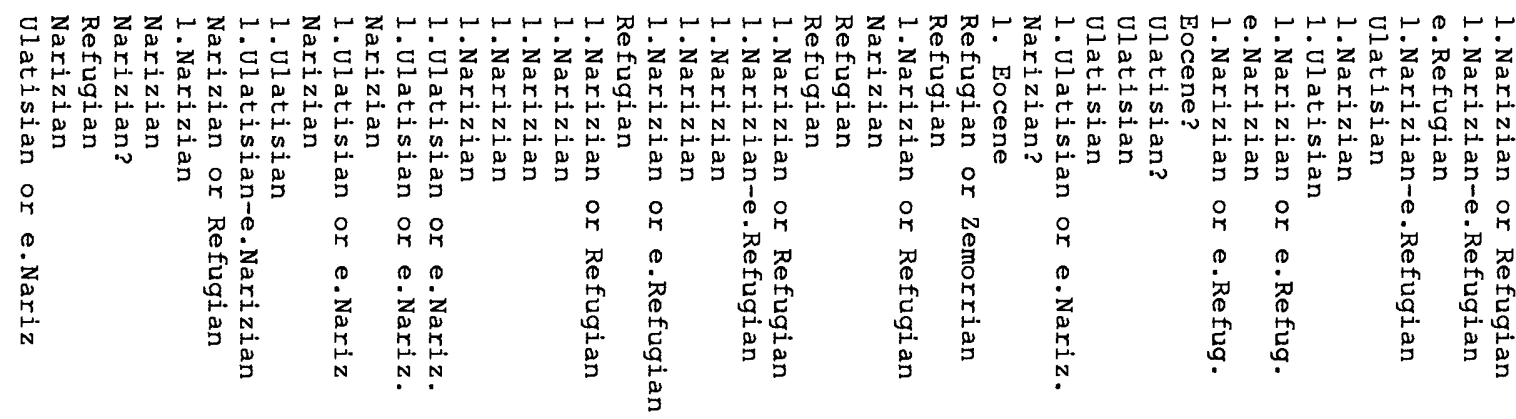

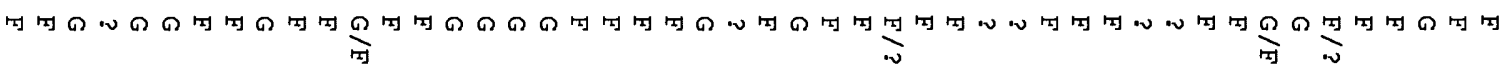

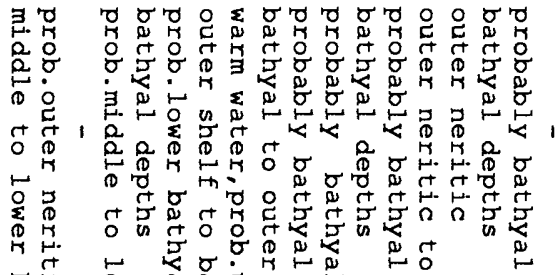

应

空

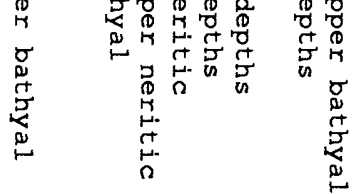

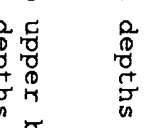

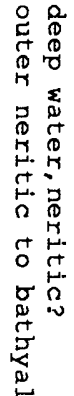

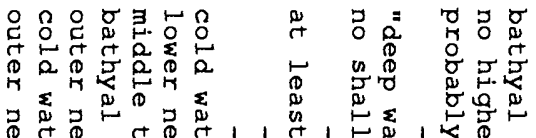

告.

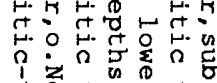

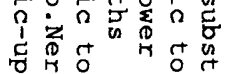

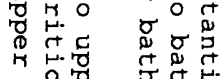

$\sigma$ 管

造造怘

苾客最

$\sim \stackrel{\sim}{\circ}$

点

\begin{tabular}{|c|c|}
\hline $\begin{array}{l}0 \\
0 \\
0 \\
0 \\
0 \\
0 \\
\sigma \\
0 \\
0\end{array}$ & 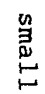 \\
\hline 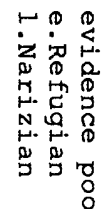 & $\begin{array}{l}0 . \\
0 . \\
0 \\
0 \\
0 \\
0 \\
0 \\
0 \\
0 \\
0 \\
0 \\
0\end{array}$ \\
\hline
\end{tabular}

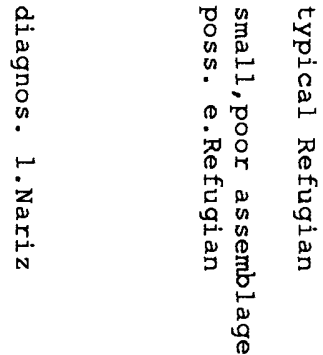

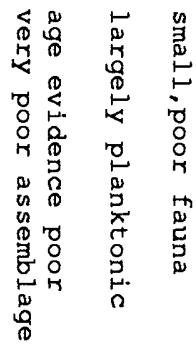

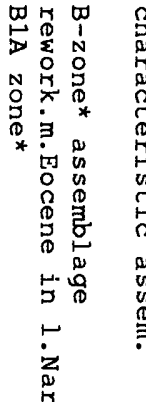

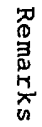




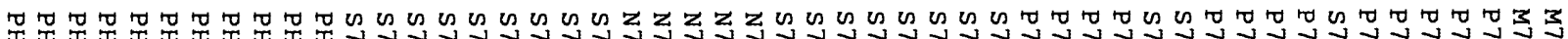

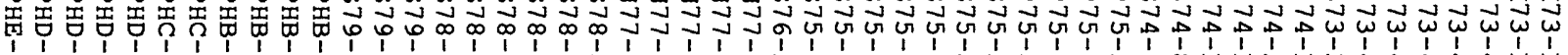

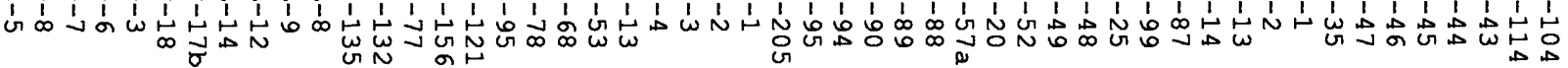

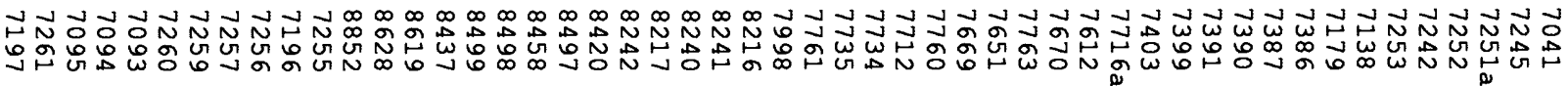

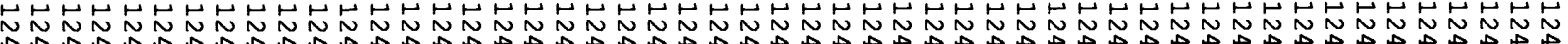

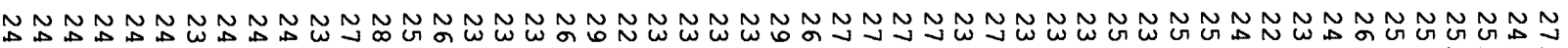

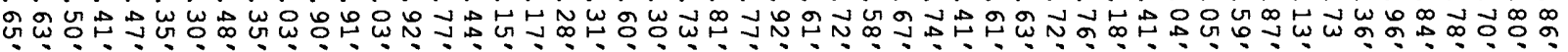

$\vec{\infty} \vec{\infty} \vec{\infty} \vec{\infty} \vec{\infty} \vec{\infty} \vec{\infty} \vec{\infty} \vec{\infty} \vec{\infty} \vec{\infty} \vec{\infty} \vec{\infty} \vec{\infty} \vec{\infty} \vec{\infty} \vec{\infty} \vec{\infty} \vec{\infty} \vec{\infty} \vec{\infty} \vec{\infty} \vec{\infty} \vec{\infty} \vec{\infty} \vec{\infty} \vec{\infty} \vec{\infty} \vec{\infty} \vec{\infty} \vec{\infty} \vec{\infty} \vec{\infty} \vec{\infty} \vec{\infty} \vec{\infty} \vec{\infty} \vec{\infty} \vec{\infty} \vec{\infty} \vec{\infty} \vec{\infty} \vec{\infty} \vec{\infty} \vec{\infty} \vec{\infty} \vec{\infty} \vec{\infty} \vec{\infty} \vec{\infty} \vec{\infty} \vec{\infty}$

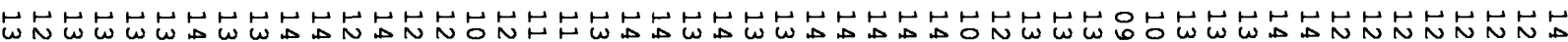

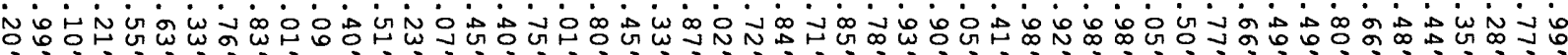

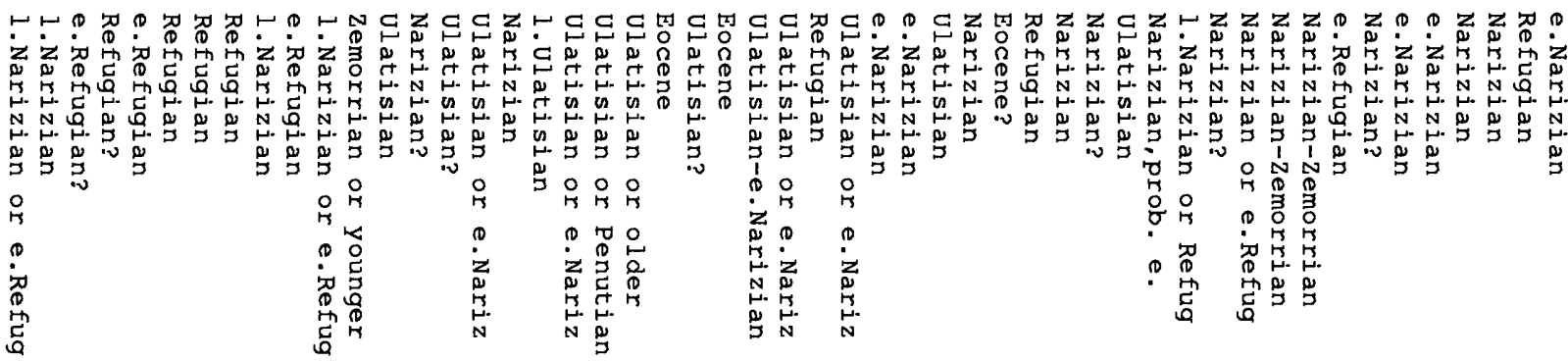

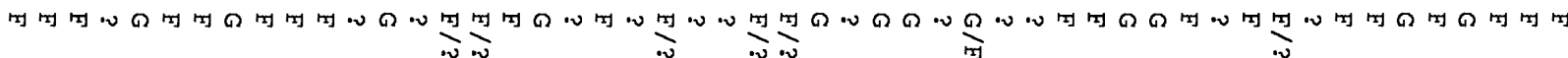

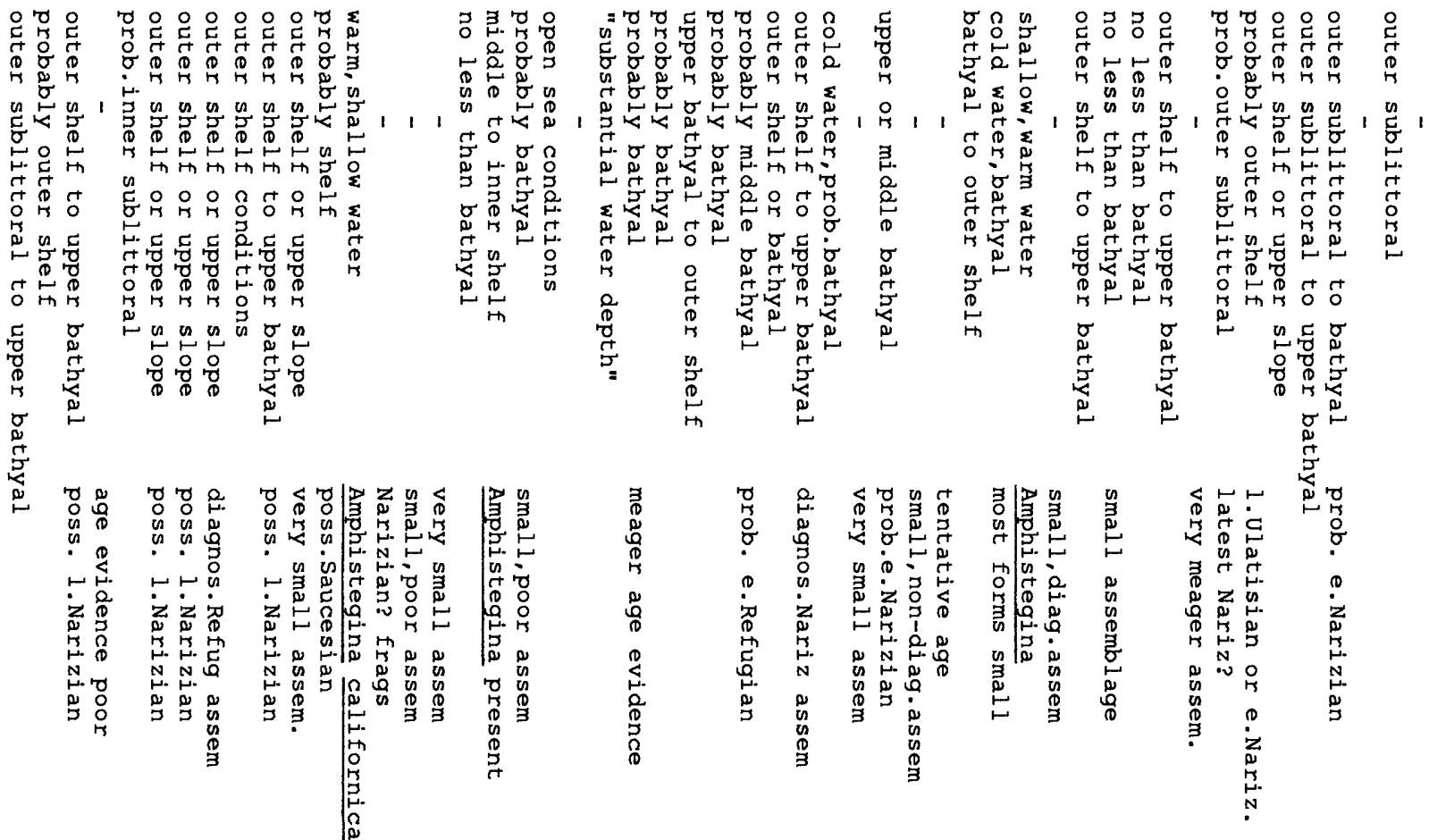




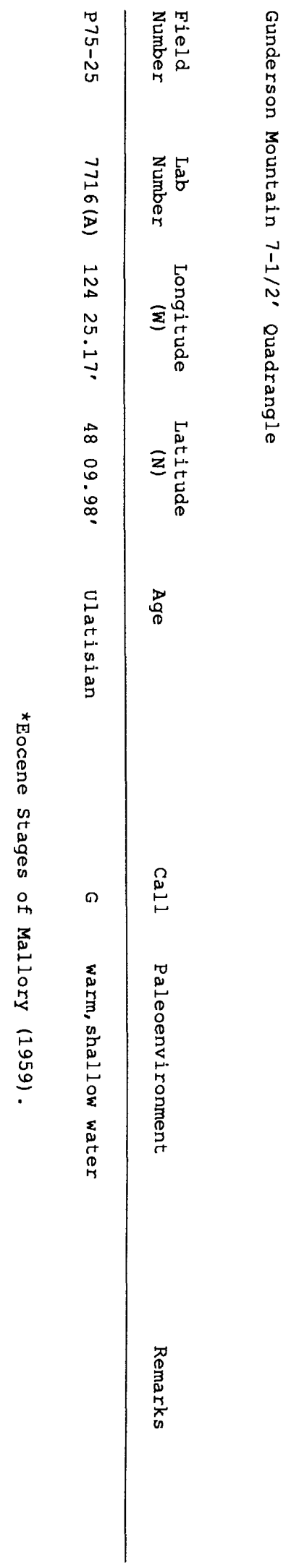

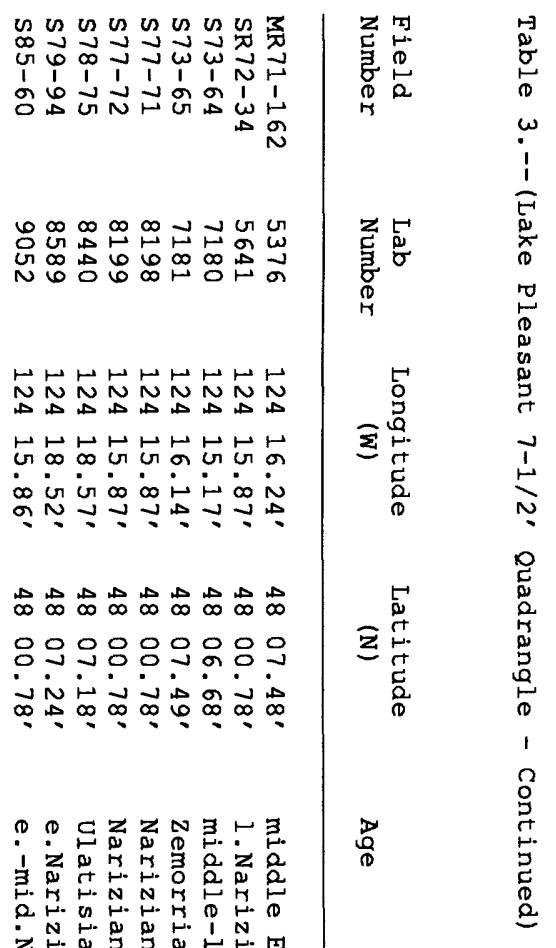

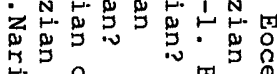

숭요

운웅

年

4.

$\stackrel{\Omega}{\varrho}$

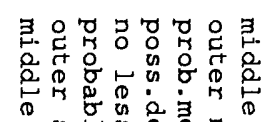

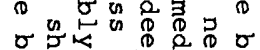

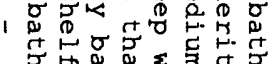

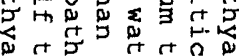

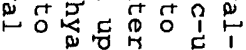

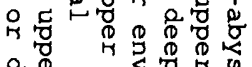

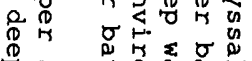

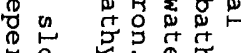

串 名 品

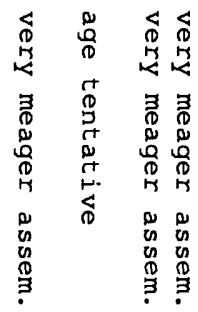

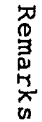




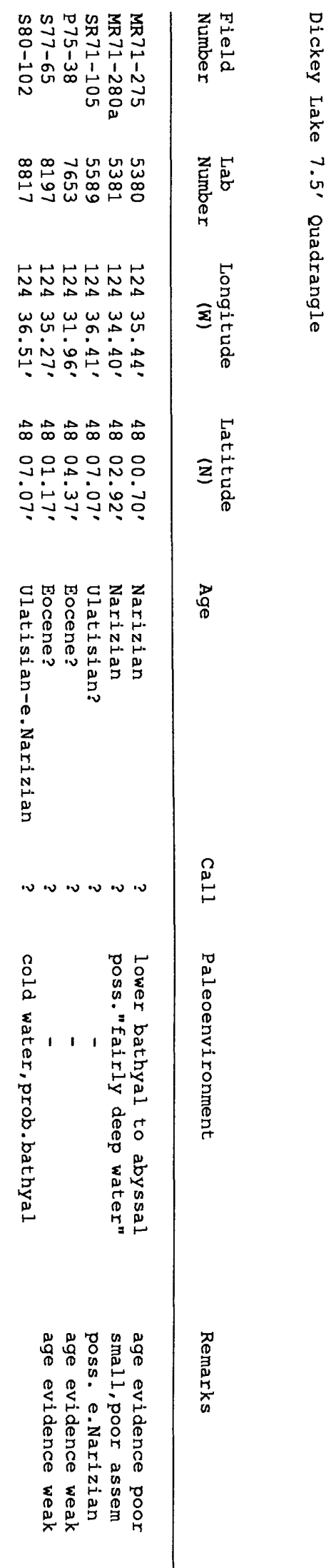

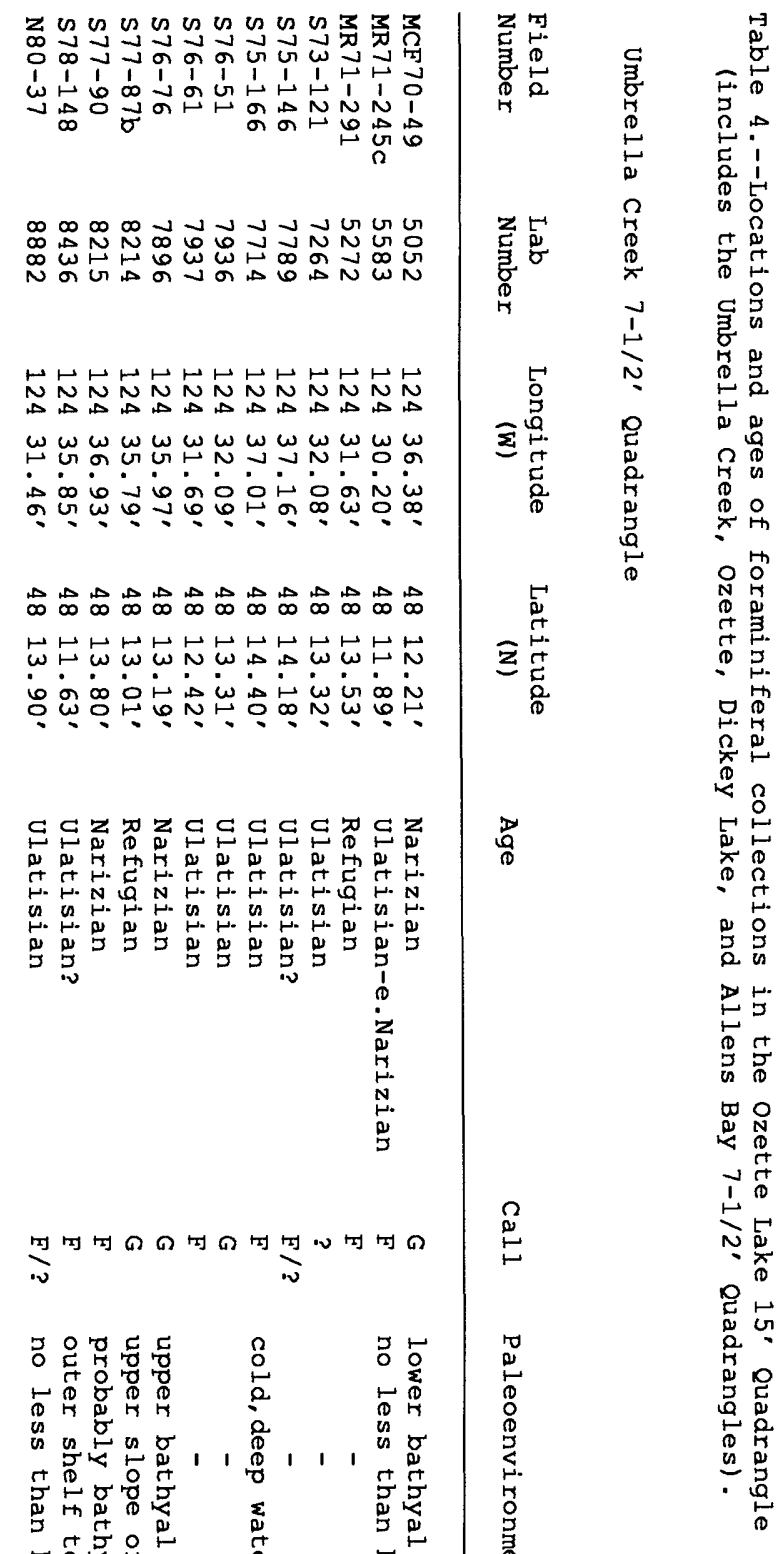

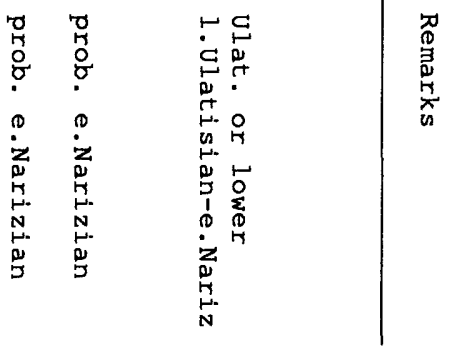




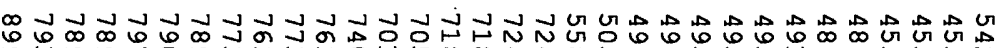

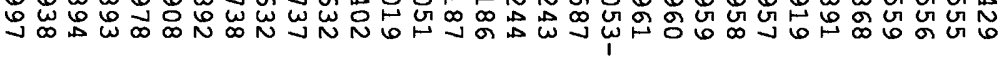

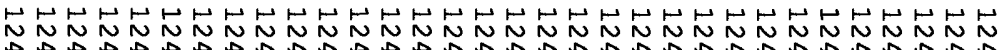

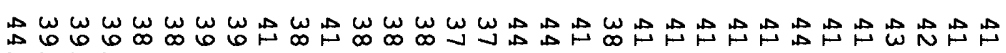

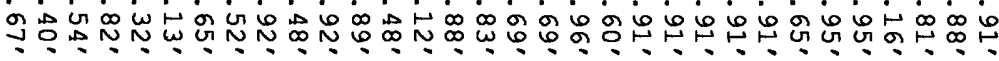

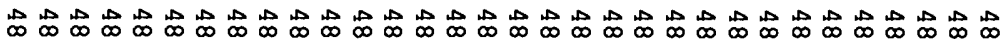

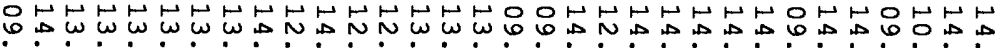

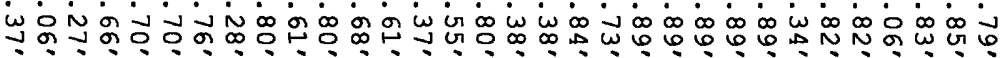

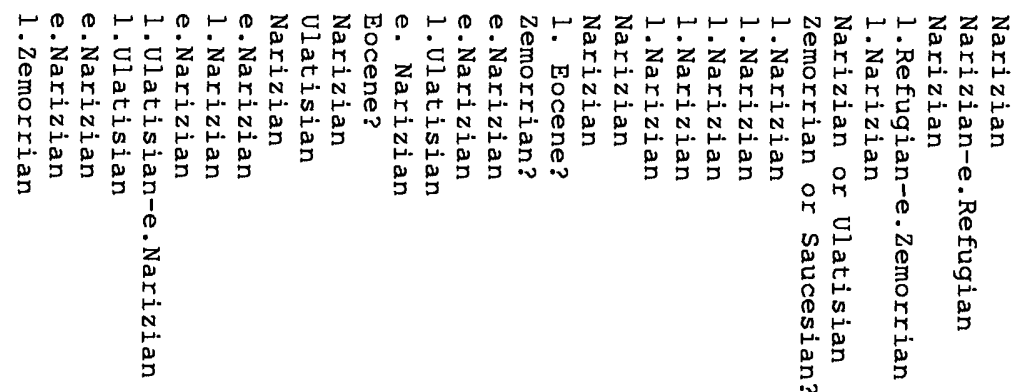

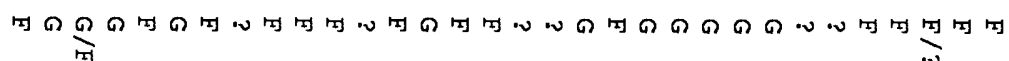

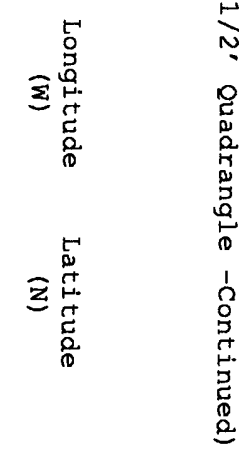

蛋

$\stackrel{2}{\stackrel{2}{⺊}}$

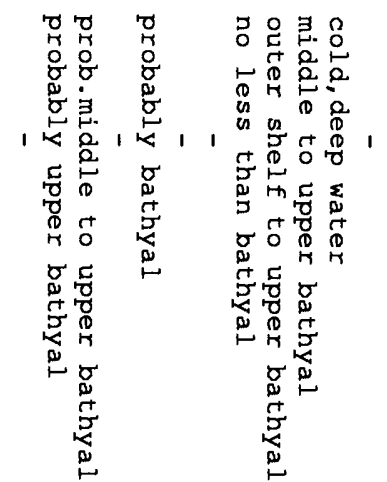

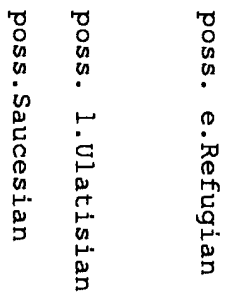

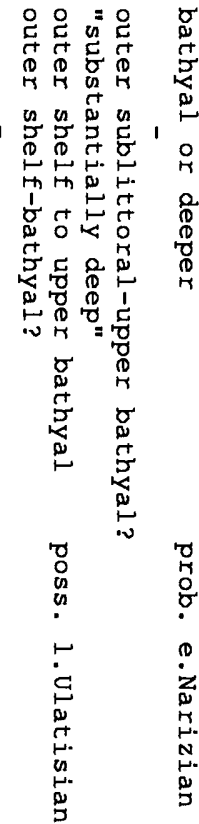

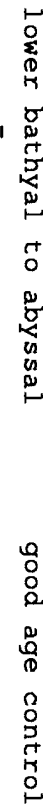

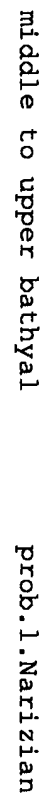




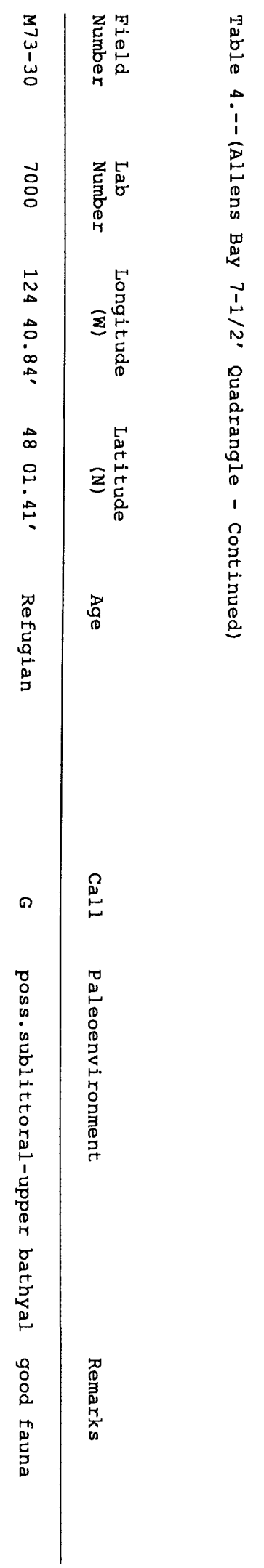

